\title{
Theory of the interaction forces and the radiative heat transfer between moving bodies
}

\author{
A. I. Volokitin ${ }^{1,2, *}$ and B. N. J. Persson ${ }^{1}$ \\ ${ }^{1}$ Institut für Festkörperforschung, Forschungszentrum Jülich, Jülich D-52425, Germany \\ ${ }^{2}$ Povolzhskaya State Academy of Telecommunication and Informatics, 443010 Samara, Russia
}

(Received 6 July 2008; published 30 October 2008)

\begin{abstract}
Within the framework of unified approach we study the Casimir-Lifshitz interaction, the van der Waals friction force, and the radiative heat transfer at nonequilibrium conditions when the interacting bodies are at different temperatures and when they move relative to each other with the arbitrary velocity $V$. The analysis is focused on the surface-surface and surface-particle configurations. We show that relativistic effects give rise to a mixing of the contributions from the electromagnetic waves with different polarization to the heat transfer and the interaction forces. We find that these effects are of the order $(V / c)^{2}$. The limiting case when one of the bodies is sufficiently rarefied gives the heat transfer and the interaction forces between a moving small particle and a surface. We also calculate the friction force acting on a particle moving with an arbitrary velocity relative to the black body radiation.
\end{abstract}

DOI: 10.1103/PhysRevB.78.155437

PACS number(s): 68.35.Af, 65.80.+n

\section{INTRODUCTION}

All bodies are surrounded by a fluctuating electromagnetic field due to the thermal and quantum fluctuations of the charge and current density inside the bodies. Outside the bodies this fluctuating electromagnetic field exists partly in the form of propagating electromagnetic waves and partly in the form of evanescent waves. The theory of the fluctuating electromagnetic field was developed by Rytov. ${ }^{1-3}$ A great variety of phenomena such as Casimir-Lifshitz forces, ${ }^{4}$ nearfield radiative heat transfer, ${ }^{5}$ and friction forces ${ }^{6}$ can be described using this theory.

Lifshitz ${ }^{4}$ used the Rytov's theory to formulate a very general theory of the dispersion interaction in the framework of the statistical physics and macroscopic electrodynamics. The Lifshitz theory provides a common tool to deal with dispersive forces in different field of science (physics, biology, and chemistry) and technology.

The Lifshitz theory is formulated for systems at thermal equilibrium. At present there is an interest in the study of systems out of the thermal equilibrium (see Ref. 7 and reference therein). The principal interest in the study of systems out of thermal equilibrium is connected to the possibility of tuning the interaction in both strength and sign. ${ }^{89}$ Such systems also give a way to explore the role of thermal fluctuations usually masked at thermal equilibrium by the $T=0 \mathrm{~K}$ component, which dominates the interaction up to very large distances, where the actual total force results in to be very small. The Casimir-Lifshitz force was measured at very large distances, and it was shown that the thermal effects of the Casimir-Lifshitz interaction are in agreement with the theoretical prediction. ${ }^{8}$ This measurement was done out of thermal equilibrium, where thermal effects are stronger.

Further thermal nonequilibrium effects were explored by Polder and Van Hove, ${ }^{5}$ who calculated the heat flux between two parallel plates. At present there is an increasing interest for studying near-field radiative heat transfer ${ }^{10-15}$ which is connected with the development of a near-field scanning thermal microscope. ${ }^{16}$ The existing studies are limited mostly by the case when the interacting bodies have different temperatures but they are at rest. For recent review of nearfield radiative heat transfer between bodies, which are at rest, see Refs. 17 and 18.

Other nonequilibrium effects are realized for bodies moving relative to each other. In Ref. 6 we used the dynamical modification of the Lifshitz theory to calculate the friction force between two plane parallel surfaces in relative motion with velocity $V$. The calculation of the van der Waals friction is more complicated than of the Casimir-Lifshitz force and the radiative heat transfer because it requires the determination of the electromagnetic field between moving boundaries. The solution can be found by writing the boundary conditions on the surface of each body in the rest reference frame of this body. The relation between the electromagnetic fields in the different reference frames is determined by the Lorenz transformation. In Ref. 6 the electromagnetic field in the vacuum gap between the bodies was calculated to linear order in $V / c$. It was shown that linear terms in the electromagnetic field give the contribution to the friction force of the order $(V / c)^{2}$. Thus, these linear terms were neglected in Ref. 6 and the resulting formula for friction force is accurate to $\operatorname{order}(V / c)^{2}$. The same approximation was used in Ref. 19 to calculate the frictional drag between quantum wells and in Refs. 20 and 21 to calculate the friction force between plane parallel surfaces in normal relative motion. For a recent review of the van der Waals friction, see Ref. 18.

In this paper within the framework of unified approach we study the Casimir-Lifshitz interaction, the van der Waals friction force, and the radiative heat transfer at nonequilibrium conditions when the interacting bodies are at different temperatures and when they move relative to each other with the arbitrary velocity $V$. Our study is focused on the surfacesurface and surface-particle configurations. In comparison with previous studies we consider more general nonequilibrium conditions. In the existing literature the CasimirLifshitz interaction and the radiative heat transfer for the surface-surface configuration were studied only for the systems out of the thermal equilibrium., ${ }^{7,17,18}$ The van der Waals friction is studied for this configuration only for systems at thermal equilibrium. ${ }^{18}$ In Sec. II we calculate the fluctuating electromagnetic field in the vacuum gap between two plane 


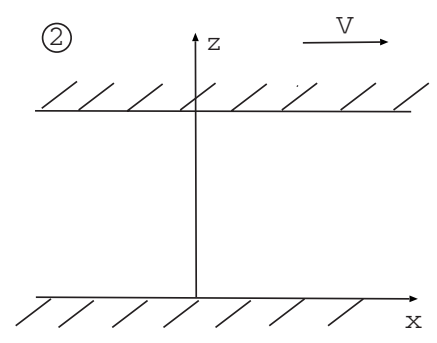

(1)

FIG. 1. Two semi-infinite bodies with plane parallel surfaces separated by a distance $d$. The upper solids moves parallel to other with velocity $V$.

parallel surfaces, moving in parallel relative to each other. In comparison with previous calculations ${ }^{6,19-21}$ we do not make any approximation in the Lorentz transformation of the electromagnetic field by means of which we can determine the field in one inertial reference frame, knowing the same field in another reference frame. Thus, our solution of the electromagnetic problem is exact. Knowing the electromagnetic field we calculate the stress tensor and the Poynting vector, which determines the interaction force and the heat transfer, respectively. We calculate the friction force, the conservative force, and the radiative heat transfer in Secs. III-V, respectively. Upon going to the limit when one of the bodies is rarefied we obtain the interaction force and the heat transfer for a small particle-surface configuration. In Sec. VI we calculate the friction force on a small particle moving relative to black body radiation. The same problem was considered in Ref. 22. In comparison with this study our treatment is relativistic and we take into account the contribution not only from the electric dipole moment but also from the magnetic moment of the particle. Recently ${ }^{23}$ it was shown that the magnetic moment gives the most important contribution to the near-field radiative heat transfer for metallic particles. The same is true for the friction force. The conclusions are given in Sec. VII.

\section{CALCUlation OF THE FLUCTUATING ELECTROMAGNETIC FIELD}

We consider two semi-infinite solids having flat parallel surfaces separated by a distance $d$ and moving with velocity $V$ relative to each other (see Fig. 1). We introduce the two coordinate systems $K$ and $K^{\prime}$ with coordinate axes $x y z$ and $x^{\prime} y^{\prime} z^{\prime}$. In the $K$ system body $\mathbf{1}$ is at rest while body $\mathbf{2}$ is moving with the velocity $V$ along the $x$ axis (the $x y$ and $x^{\prime} y^{\prime}$ planes are in the surface of body $\mathbf{1}$, the $x$ and $x^{\prime}$ axes have the same direction, and the $z$ and $z^{\prime}$ axes point toward body 2). In the $K^{\prime}$ system body $\mathbf{2}$ is at rest while body $\mathbf{1}$ is moving with velocity $-V$ along the $x$ axis. Since the system is translational invariant in the $\mathbf{x}=(x, y)$ plane, the electromagnetic field can be represented by the Fourier integrals

$$
\mathbf{E}(\mathbf{x}, z, t)=\int_{-\infty}^{\infty} d \omega \int \frac{d^{2} q}{(2 \pi)^{2}} e^{i \mathbf{q} \cdot \mathbf{x}-i \omega t} \mathbf{E}(\mathbf{q}, \omega, z),
$$

$$
\mathbf{B}(\mathbf{x}, z, t)=\int_{-\infty}^{\infty} d \omega \int \frac{d^{2} q}{(2 \pi)^{2}} e^{i \mathbf{q} \cdot \mathbf{x}-i \omega t} \mathbf{B}(\mathbf{q}, \omega, z),
$$

where $\mathbf{E}$ and $\mathbf{B}$ are the electric and magnetic induction field, respectively, and $\mathbf{q}$ is the two-dimensional wave vector in $x y$ plane. After Fourier transformation it is convenient to decompose the electromagnetic field into $s$ - and $p$-polarized components. For the $p$ - and $s$-polarized electromagnetic waves the electric field $\mathbf{E}(\mathbf{q}, \omega, z)$ is in plane of incidence and perpendicular to that plane, respectively. In the vacuum gap between the bodies the electric field $\mathbf{E}(\mathbf{q}, \omega, z)$ and the magnetic induction field $\mathbf{B}(\mathbf{q}, \omega, z)$ can be written in the form

$$
\mathbf{E}(\mathbf{q}, \omega, z)=\left(v_{s} \hat{n}_{s}+v_{p} \hat{n}_{p}^{+}\right) e^{i k_{z} z}+\left(w_{s} \hat{n}_{s}+w_{p} \hat{n}_{p}^{-}\right) e^{-i k_{z} z},
$$

$$
\begin{aligned}
\mathbf{B}(\mathbf{q}, \omega, z)= & \frac{c}{\omega}\left\{\left[\left(\mathbf{k}^{+} \times \hat{n}_{s}\right) v_{s}+\left(\mathbf{k}^{+} \times \hat{n}_{p}^{+}\right) v_{p}\right] e^{i k_{z} z}\right. \\
& \left.+\left[\left(\mathbf{k}^{-} \times \hat{n}_{s}\right) w_{s}+\left(\mathbf{k}^{-} \times \hat{n}_{p}^{-}\right) w_{p}\right] e^{-i k_{z} z}\right\},
\end{aligned}
$$

where $\quad \mathbf{k}^{ \pm}=\mathbf{q} \pm \hat{z} k_{z}, \quad k_{z}=\left[(\omega / c)^{2}-q^{2}\right]^{1 / 2}, \quad \hat{n}_{s}=[\hat{z} \times \hat{q}]$ $=\left(-q_{y}, q_{x}, 0\right) / q, \hat{n}_{p}^{ \pm}=\left[\hat{k}^{ \pm} \times \hat{n}_{s}\right]=\left(\mp q_{x} k_{z}, \mp q_{y} k_{z}, q^{2}\right) /(k q)$, and $k=\omega / c$. At the surfaces of the bodies the amplitude of the outgoing electromagnetic wave must be equal to the amplitude of the reflected wave plus the amplitude of the radiated wave. Thus, the boundary conditions for the electromagnetic field at $z=0$ in the $K$ reference frame can be written in the form

$$
v_{p(s)}=R_{1 p(s)}(\omega, q) w_{p(s)}+E_{1 p(s)}^{f}(\omega, q),
$$

where $R_{1 p(s)}(\omega)$ is the reflection amplitude for surface 1 for the $p(s)$-polarized electromagnetic field and $E_{1 p(s)}^{f}(\omega)$ is the amplitude of the fluctuating electric field radiated by body 1 for a $p(s)$-polarized wave. In the $K^{\prime}$ reference frame the electric field can be written in the form

$$
\mathbf{E}^{\prime}\left(\mathbf{q}^{\prime}, \omega^{\prime}, z\right)=\left(v_{s}^{\prime} \hat{n}_{s}^{\prime}+v_{p}^{\prime} \hat{n}_{p}^{\prime+}\right) e^{i k_{z} z}+\left(w_{s}^{\prime} \hat{n}_{s}^{\prime}+w_{p}^{\prime} \hat{n}_{p}^{\prime-}\right) e^{-i k_{z} z},
$$

where $\mathbf{q}^{\prime}=\left(q_{x}^{\prime}, q_{y}, 0\right), \quad q_{x}^{\prime}=\left(q_{x}-\beta k\right) \gamma, \quad \omega^{\prime}=\left(\omega-V q_{x}\right) \gamma, \quad \gamma$ $=1 / \sqrt{1-\beta^{2}}, \quad \beta=V / c, \quad \hat{n}_{s}^{\prime}=\left(-q_{y}, q_{x}^{\prime}, 0\right) / q^{\prime}, \quad \hat{n}_{p}^{\prime \pm}$ $=\left(\mp q_{x}^{\prime} k_{z}, \mp q_{y} k_{z}, q^{\prime 2}\right) /\left(k^{\prime} q^{\prime}\right)$, and

$$
q^{\prime}=\gamma \sqrt{q^{2}-2 \beta k q_{x}+\beta^{2}\left(k^{2}-q_{y}^{2}\right)} .
$$

The boundary conditions at $z=d$ in the $K^{\prime}$ reference frame can be written in a form similar to Eq. (5), as

$$
w_{p(s)}^{\prime}=e^{2 i k_{z} d} R_{2 p(s)}\left(\omega^{\prime}, q^{\prime}\right) v_{p(s)}^{\prime}+e^{i k_{z} d} E_{2 p(s)}^{\prime f}\left(\omega^{\prime}, q^{\prime}\right),
$$

where $R_{2 p(s)}(\omega)$ is the reflection amplitude for surface 2 for $p(s)$-polarized electromagnetic field and $E_{2 p(s)}^{\prime f}(\omega)$ is the amplitude of the fluctuating electric field radiated by body 2 for a $p(s)$-polarized wave. A Lorentz transformation for the electric field gives

$$
E_{x}^{\prime}=E_{x}, E_{y}^{\prime}=\left(E_{y}-\beta B_{z}\right) \gamma, E_{z}^{\prime}=\left(E_{z}+\beta B_{y}\right) \gamma .
$$

Using Eqs. (3), (4), (6), and (8) we get

$$
v_{p}^{\prime}=\frac{k^{\prime} \gamma}{k q q^{\prime}}\left[-\beta k_{z} q_{y} v_{s}+\left(q^{2}-\beta k q_{x}\right) v_{p}\right],
$$




$$
\begin{gathered}
w_{p}^{\prime}=\frac{k^{\prime} \gamma}{k q q^{\prime}}\left[\beta k_{z} q_{y} w_{s}+\left(q^{2}-\beta k q_{x}\right) w_{p}\right], \\
v_{s}^{\prime}=\frac{k^{\prime} \gamma}{k q q^{\prime}}\left[\beta k_{z} q_{y} v_{p}+\left(q^{2}-\beta k q_{x}\right) v_{s}\right], \\
w_{s}^{\prime}=\frac{k^{\prime} \gamma}{k q q^{\prime}}\left[-\beta k_{z} q_{y} w_{p}+\left(q^{2}-\beta k q_{x}\right) w_{s}\right] .
\end{gathered}
$$

Substituting Eqs. (9)-(12) in Eq. (7) and using Eq. (5) we get

$$
\begin{aligned}
& \left(q^{2}-\beta k q_{x}\right) D_{p p} w_{p}+\beta k_{z} q_{y} D_{s p} w_{s} \\
& =e^{2 i k_{z} d} R_{2 p}^{\prime}\left[\left(q^{2}-\beta k q_{x}\right) E_{1 p}^{f}-\beta k_{z} q_{y} E_{1 s}^{f}\right]+\frac{k q q^{\prime}}{k^{\prime} \gamma} e^{i k_{z} d} E_{2 p}^{\prime f}, \\
& \left(q^{2}-\beta k q_{x}\right) D_{s s} w_{s}-\beta k_{z} q_{y} D_{p s} w_{p} \\
& =e^{2 i k_{z} d} R_{2 s}^{\prime}\left[\left(q^{2}-\beta k q_{x}\right) E_{1 s}^{f}+\beta k_{z} q_{y} E_{1 p}^{f}\right]+\frac{k q q^{\prime}}{k^{\prime} \gamma} e^{i k_{z} d} E_{2 s}^{\prime f},
\end{aligned}
$$

where

$$
\begin{aligned}
& D_{p p}=1-e^{2 i k_{z} d} R_{1 p} R_{2 p}^{\prime}, \quad D_{s s}=1-e^{2 i k_{z} d} R_{1 s} R_{2 s}^{\prime}, \\
& D_{s p}=1+e^{2 i k_{z} d} R_{1 s} R_{2 p}^{\prime}, \quad D_{p s}=1+e^{2 i k_{z} d} R_{1 p} R_{2 s}^{\prime},
\end{aligned}
$$

$R_{2 p(s)}^{\prime}=R_{2 p(s)}\left(\omega^{\prime}, q^{\prime}\right)$. From Eqs. (13), (14), and (5) we get

$$
\begin{aligned}
w_{p}= & \left\{\left[\left(q^{2}-\beta k q_{x}\right)^{2} R_{2 p}^{\prime} D_{s s}-\beta^{2} k_{z}^{2} q_{y}^{2} R_{2 s}^{\prime} D_{s p}\right] E_{1 p}^{f} e^{2 i k_{z} d}\right. \\
& -\beta k_{z} q_{y}\left(q^{2}-\beta k q_{x}\right)\left(R_{2 p}^{\prime}+R_{2 s}^{\prime}\right) E_{1 s}^{f} e^{2 i k_{z} d} \\
& \left.+\frac{k q q^{\prime}}{k^{\prime} \gamma}\left[\left(q^{2}-\beta k q_{x}\right) D_{s s} E_{2 p}^{\prime f}-\beta k_{z} q_{y} D_{s p} E_{2 s}^{\prime f}\right] e^{i k_{z} d}\right\} \Delta^{-1},
\end{aligned}
$$

$$
\begin{aligned}
v_{p}= & \left\{\left[\left(q^{2}-\beta k q_{x}\right)^{2} D_{s s}+\beta^{2} k_{z}^{2} q_{y}^{2} D_{s p}\right] E_{1 p}^{f}-\beta k_{z} q_{y}\left(q^{2}\right.\right. \\
& \left.-\beta k q_{x}\right) R_{1 p}\left(R_{2 p}^{\prime}+R_{2 s}^{\prime}\right) e^{2 i k_{z} d} E_{1 s}^{f}+\frac{k q q^{\prime}}{k^{\prime} \gamma} R_{1 p}\left[\left(q^{2}\right.\right. \\
& \left.\left.\left.-\beta k q_{x}\right) D_{s s} E_{2 p}^{\prime f}-\beta k_{z} q_{y} D_{s p} E_{2 s}^{\prime f}\right] e^{i k_{z} d}\right\} \Delta^{-1}, \\
w_{s}= & \left\{\left[\left(q^{2}-\beta k q_{x}\right)^{2} R_{2 s}^{\prime} D_{p p}-\beta^{2} k_{z}^{2} q_{y}^{2} R_{2 p}^{\prime} D_{p s}\right] E_{1 s}^{f} e^{2 i k_{z} d}\right. \\
+ & \beta k_{z} q_{y}\left(q^{2}-\beta k q_{x}\right)\left(R_{2 p}^{\prime}+R_{2 s}^{\prime}\right) E_{1 p}^{f} e^{2 i k_{z} d} \\
+ & \left.\frac{k q q^{\prime}}{k^{\prime} \gamma}\left[\left(q^{2}-\beta k q_{x}\right) D_{p p} E_{2 s}^{\prime f}+\beta k_{z} q_{y} D_{p s} E_{2 p}^{\prime f}\right] e^{i k_{z} d}\right\} \Delta^{-1},
\end{aligned}
$$

$$
\begin{aligned}
v_{s}= & \left\{\left[\left(q^{2}-\beta k q_{x}\right)^{2} D_{p p}+\beta^{2} k_{z}^{2} q_{y}^{2} D_{p s}\right] E_{1 s}^{f}+\beta k_{z} q_{y}\left(q^{2}\right.\right. \\
& \left.-\beta k q_{x}\right) R_{1 p}\left(R_{2 p}^{\prime}+R_{2 s}^{\prime}\right) e^{2 i k_{z} d} E_{1 p}^{f}+\frac{k q q^{\prime}}{k^{\prime} \gamma} R_{1 s}\left[\left(q^{2}\right.\right. \\
& \left.\left.\left.-\beta k q_{x}\right) D_{p p} E_{2 s}^{\prime f}+\beta k_{z} q_{y} D_{p s} E_{2 p}^{\prime f}\right] e^{i k_{z} d}\right\} \Delta^{-1},
\end{aligned}
$$

where

$$
\Delta=\left(q^{2}-\beta k q_{x}\right)^{2} D_{s s} D_{p p}+\beta^{2} k_{z}^{2} q_{y}^{2} D_{p s} D_{s p} .
$$

The fundamental characteristic of the fluctuating electromagnetic field is the correlation function, determining the average product of amplitudes $E_{p(s)}^{f}(\mathbf{q}, \omega)$. According to the general theory of the fluctuating electromagnetic field (see for example Ref. 18),

$$
\begin{aligned}
\left\langle\left|E_{p(s)}^{f}(\mathbf{q}, \omega)\right|^{2}\right\rangle= & \frac{\hbar \omega^{2}}{2 c^{2}\left|k_{z}\right|^{2}}\left(n(\omega)+\frac{1}{2}\right)\left[\left(k_{z}+k_{z}^{*}\right)\left(1-\left|R_{p(s)}\right|^{2}\right)\right. \\
& \left.+\left(k_{z}-k_{z}^{*}\right)\left(R_{p(s)}^{*}-R_{p(s)}\right)\right]
\end{aligned}
$$

where $\langle\ldots\rangle$ denotes statistical average over the random field. We note that $k_{z}$ is real for $q<\omega / c$ (propagating waves) and purely imaginary for $q>\omega / c$ (evanescent waves). The BoseEinstein factor

$$
n(\omega)=\frac{1}{e^{\hbar \omega / k_{B} T}-1} .
$$

Thus for $q<\omega / c$ and $q>\omega / c$ the correlation functions are determined by the first and the second terms in Eq. (19), respectively.

\section{CALCULATION OF THE FRICTION FORCE}

The force which acts on the surface of body $\mathbf{1}$ can be calculated from the Maxwell stress tensor $\sigma_{i j}$, evaluated at $z=0$,

$$
\begin{aligned}
\sigma_{i j}= & \frac{1}{4 \pi} \int_{0}^{\infty} d \omega \int \frac{d^{2} q}{(2 \pi)^{2}}\left[\left\langle E_{i} E_{j}^{*}\right\rangle+\left\langle E_{i}^{*} E_{j}\right\rangle+\left\langle B_{i} B_{j}^{*}\right\rangle+\left\langle B_{i}^{*} B_{j}\right\rangle\right. \\
& \left.-\delta_{i j}\left(\left\langle\mathbf{E} \cdot \mathbf{E}^{*}\right\rangle+\left\langle\mathbf{B} \cdot \mathbf{B}^{*}\right\rangle\right)\right]_{z=0} .
\end{aligned}
$$

Using Eqs. (3) and (4) for the $x$ component of the force we get

$$
\begin{aligned}
\sigma_{x z}= & \frac{1}{4 \pi} \int_{0}^{\infty} d \omega \int \frac{d^{2} q}{(2 \pi)^{2}} \frac{q_{x}}{k^{2}}\left[( k _ { z } + k _ { z } ^ { * } ) \left(\left\langle\left|w_{p}\right|^{2}\right\rangle+\left\langle\left|w_{s}\right|^{2}\right\rangle\right.\right. \\
& \left.\left.-\left\langle\left|v_{p}\right|^{2}\right\rangle-\left\langle\left|v_{s}\right|^{2}\right\rangle\right)+\left(k_{z}-k_{z}^{*}\right)\left\langle w_{p} v_{p}^{*}+w_{s} v_{s}^{*}-\text { c.c. }\right\rangle\right] .
\end{aligned}
$$

Substituting Eqs. (15)-(18) for the amplitudes of the electromagnetic field in Eq. (21), and performing averaging over the fluctuating electromagnetic field with the help of Eq. (19), we get the $x$ component of the force 


$$
\begin{aligned}
& F_{x}=\sigma_{x z}=\frac{\hbar}{8 \pi^{3}} \int_{0}^{\infty} d \omega \int_{q<\omega / c} d^{2} q \frac{q_{x}}{|\Delta|^{2}}\left[\left(q^{2}-\beta k q_{x}\right)^{2}+\beta^{2} k_{z}^{2} q_{y}^{2}\right] \\
& \times\left[\left(q^{2}-\beta k q_{x}\right)^{2}\left(1-\left|R_{1 p}\right|^{2}\right)\left(1-\left|R_{2 p}^{\prime}\right|^{2}\right)\left|D_{s s}\right|^{2}\right. \\
& +\beta^{2} k_{z}^{2} q_{y}^{2}\left(1-\left|R_{1 p}\right|^{2}\right)\left(1-\left|R_{2 s}^{\prime}\right|^{2}\right)\left|D_{s p}\right|^{2} \\
& +(p \leftrightarrow s)]\left[n_{2}\left(\omega^{\prime}\right)-n_{1}(\omega)\right] \\
& +\frac{\hbar}{2 \pi^{3}} \int_{0}^{\infty} d \omega \int_{q>\omega / c} d^{2} q \frac{q_{x}}{|\Delta|^{2}}\left[\left(q^{2}-\beta k q_{x}\right)^{2}\right. \\
& \left.+\beta^{2} k_{z}^{2} q_{y}^{2}\right] e^{-2\left|k_{z}\right| d[}\left[\left(q^{2}-\beta k q_{x}\right)^{2} \operatorname{Im} R_{1 p} \quad \operatorname{Im} R_{2 p}^{\prime}\left|D_{s s}\right|^{2}\right. \\
& \left.-\beta^{2} k_{z}^{2} q_{y}^{2} \operatorname{Im} R_{1 p} \operatorname{Im} R_{2 s}^{\prime}\left|D_{s p}\right|^{2}+(p \leftrightarrow s)\right]\left[n_{2}\left(\omega^{\prime}\right)\right. \\
& \left.-n_{1}(\omega)\right] \text {, }
\end{aligned}
$$

where

$$
n_{i}(\omega)=\frac{1}{e^{\hbar \omega / k_{B} T_{i}}-1},
$$

where $T_{1}$ and $T_{2}$ are the temperatures for bodies $\mathbf{1}$ and $\mathbf{2}$, respectively. The symbol $(p \leftrightarrow s)$ denotes the terms which can be obtained from the preceding terms by permutation of the indexes $p$ and $s$. The first term in Eq. (22) represents the contribution to the friction from propagating waves $(q$ $<\omega / c)$, and the second term from the evanescent waves $(q$ $>\omega / c$ ). If in Eq. (22) one neglects the terms of the order $\beta^{2}$ then the contributions from waves with $p$ and $s$ polarization will be separated. In this case Eq. (22) is reduced to the formula obtained in Ref. 6 . Thus, to the order $\beta^{2}$ the mixing of waves with different polarization can be neglected, what agrees with the results obtained in Ref. 6. At $T=0 \mathrm{~K}$ the propagating waves do not contribute to friction, but the contribution from evanescent waves is not equal to zero. Taking into account that $n(-\omega)=-1-n(\omega)$ from Eq. (22) we get friction mediated by the evanescent electromagnetic waves at zero temperature (in literature this type of friction is named as quantum friction ${ }^{24}$ ),

$$
\begin{aligned}
& F_{x}=-\frac{\hbar}{\pi^{3}} \int_{0}^{\infty} d q_{y} \int_{0}^{\infty} d q_{x} \int_{0}^{q_{x} V} d \omega \frac{q_{x}}{|\Delta|^{2}}\left[\left(q^{2}-\beta k q_{x}\right)^{2}\right. \\
& \left.+\beta^{2} k_{z}^{2} q_{y}^{2}\right] e^{-2\left|k_{z}\right| d}\left[\left(q^{2}-\beta k q_{x}\right)^{2} \operatorname{Im} R_{1 p} \operatorname{Im} R_{2 p}^{\prime}\left|D_{s s}\right|^{2}\right. \\
& \left.-\beta^{2} k_{z}^{2} q_{y}^{2} \operatorname{Im} R_{1 p} \operatorname{Im} R_{2 s}^{\prime}\left|D_{s p}\right|^{2}+(p \leftrightarrow s)\right] .
\end{aligned}
$$

Figure 2 shows the dependence of the frictional stress between semi-infinite bodies on the velocity $V$ at different separation $d$. In the calculations the Fresnel formulas for the reflection amplitude were used with the Drude permittivity $\varepsilon$ for copper. The frictional stress initially increases with velocity, reaches a maximum, and then decreases at large values of the velocity. The presence of maximum is connected with the resonances which are present in the integrand for the friction force due to the coupled plasmon-polariton of both surfaces. ${ }^{17,18}$ Doppler shift leads to the displacement of resonances relative to each other. If this displacement becomes larger than the width of resonance then this leads to the decrease in friction force when the velocity increases.

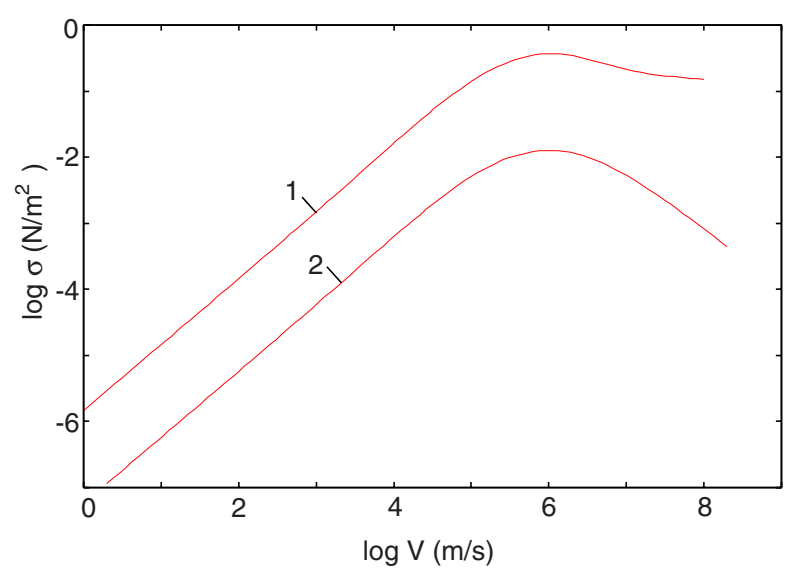

FIG. 2. (Color online) The velocity dependence of the frictional stress acting between two semi-infinite bodies at (1) $d=10 \mathrm{~nm}$ and (2) $d=100 \mathrm{~nm}$ with parameters chosen to correspond to copper $\left(\tau^{-1}=2.5 \times 10^{13} \mathrm{~s}^{-1}\right.$ and $\left.\omega_{p}=1.6 \times 10^{16} \mathrm{~s}^{-1}\right)$. (The base of the logarithm is 10 .)

The friction force acting on a small particle moving in parallel to a flat surface can be obtained from the friction between two semi-infinite bodies in the limit when one of the bodies is sufficiently rarefied. For $d \ll \lambda_{T}=c \hbar / k_{B} T$, in Eq. (22) we can neglect the first term, and in the second term we can integrate over the whole $q$ plane and put $k_{z} \approx i q$. We will assume that the rarefied body consists of small metal particles, which have the electric dipole moment and the magnetic moment. The dielectric permittivity and magnetic permeability of this body, say body $\mathbf{2}$, is close to unity, i.e., $\varepsilon_{2}$ $-1 \rightarrow 4 \pi n \alpha_{E} \ll 1$ and $\mu_{2}-1 \rightarrow 4 \pi n \alpha_{H} \ll 1$, where $n$ is the concentration of particles in body $2, \alpha_{E}$ and $\alpha_{H}$ are their electric and magnetic susceptibilities. To linear order in the concentration $n$ the reflection amplitudes are

$$
\begin{gathered}
R_{2 p}=\frac{\varepsilon_{2} k_{z}-\sqrt{\varepsilon_{2} \mu_{2} k^{2}-q^{2}}}{\varepsilon_{2} k_{z}+\sqrt{\varepsilon_{2} \mu_{2} k^{2}-q^{2}}} \approx \frac{\varepsilon_{2}-1}{\varepsilon_{2}+1} \approx 2 \pi n \alpha_{E}, \\
R_{2 s}=\frac{\mu_{2} k_{z}-\sqrt{\varepsilon_{2} \mu_{2} k^{2}-q^{2}}}{\mu_{2} k_{z}+\sqrt{\varepsilon_{2} \mu_{2} k^{2}-q^{2}}} \approx \frac{\mu_{2}-1}{\mu_{2}+1} \approx 2 \pi n \alpha_{H} .
\end{gathered}
$$

The friction force acting on a particle moving in parallel to a plane surface can be obtained as the ratio between the change of the frictional shear stress between two surfaces after displacement of body $\mathbf{2}$ by small distance $d z$, and the number of the particles in a slab with thickness $d z$,

$$
\begin{aligned}
F_{x}^{p a r t}= & \left.\frac{d \sigma_{\|}(z)}{n d z}\right|_{z=d}=\frac{2 \hbar}{\pi^{2}} \int_{0}^{\infty} d \omega \int d^{2} q \frac{q_{x} q}{q^{2}-\beta^{2} q_{y}^{2}} e^{-2 q d}\left[n_{2}\left(\omega^{\prime}\right)\right. \\
& \left.-n_{1}(\omega)\right]\left[q^{2}\left(\operatorname{Im} R_{p} \operatorname{Im} \alpha_{E}^{\prime}+\operatorname{Im} R_{s} \operatorname{Im} \alpha_{H}^{\prime}\right)\right. \\
& \left.+\beta^{2} q_{y}^{2}\left(\operatorname{Im} R_{p} \operatorname{Im} \alpha_{H}^{\prime}+\operatorname{Im} R_{s} \operatorname{Im} \alpha_{E}^{\prime}\right)\right]
\end{aligned}
$$

where $\alpha_{E(H)}^{\prime}=\alpha_{E(H)}\left(\omega^{\prime}\right)$. For a spherical particle with radius $R$ the electric and magnetic susceptibilities are given by ${ }^{25}$

$$
\alpha_{E}=R^{3} \frac{\varepsilon-1}{\varepsilon+2},
$$




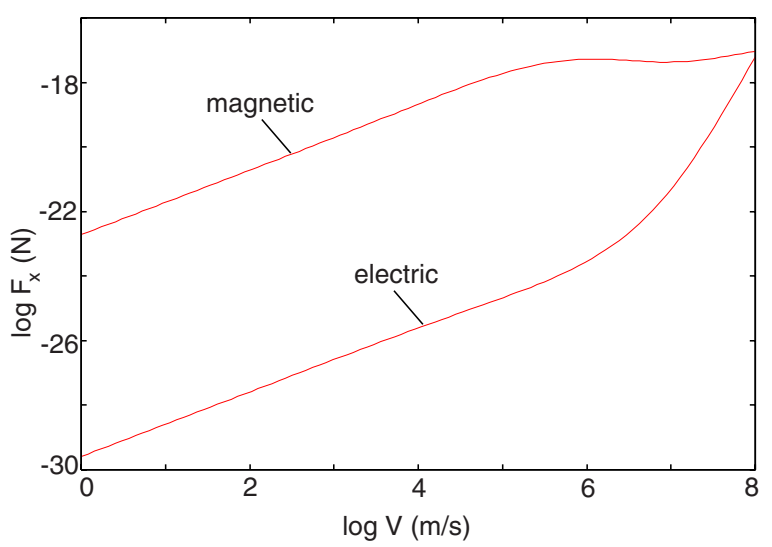

FIG. 3. (Color online) The velocity dependence of the friction force acting on small copper particle with radius $R=10 \mathrm{~nm}$ moving above a copper sample at the separation $d=20 \mathrm{~nm}$. The contributions from the electric dipole moment and the magnetic moment are shown separately. (The base of the logarithm is 10.)

$$
\alpha_{H}=-\frac{R^{3}}{2}\left[1-\frac{3}{R^{2} \kappa^{2}}+\frac{3}{R \kappa} \cot R \kappa\right],
$$

where $\kappa=k \sqrt{\varepsilon-1}$. Figure 3 shows the velocity dependence of the friction force, which acts on a small copper particle with $R=10 \mathrm{~nm}$, moving above the copper sample at $d=20 \mathrm{~nm}$. The contributions from the electric dipole and magnetic moments are shown separately. At small velocities the contribution from the magnetic moment is seven orders of magnitude larger than the contribution from the electric dipole moment.

\section{CALCULATION OF THE CONSERVATIVE FORCE BETWEEN MOVING BODIES}

The $z$ component of the force is determined by $z z$ component of the Maxwell stress tensor

$$
\begin{aligned}
\sigma_{z z}= & -\frac{1}{4 \pi} \int_{0}^{\infty} d \omega \int \frac{d^{2} q}{(2 \pi)^{2}} \frac{k_{z}}{k^{2}}\left[( k _ { z } + k _ { z } ^ { * } ) \left(\left\langle\left|w_{p}\right|^{2}\right\rangle+\left\langle\left|w_{s}\right|^{2}\right\rangle\right.\right. \\
& \left.\left.+\left\langle\left|v_{p}\right|^{2}\right\rangle+\left\langle\left|v_{s}\right|^{2}\right\rangle\right)+\left(k_{z}-k_{z}^{*}\right)\left\langle w_{p} v_{p}^{*}+w_{s} v_{s}^{*}+\text { c.c. }\right\rangle\right] .
\end{aligned}
$$

One has to subtract the infinite vacuum contribution to the force which does not depend on separation $d .^{4,26}$ Substituting Eqs. (15)-(18) into Eq. (27), and averaging over the fluctuating electromagnetic field with the help of Eq. (19), and after subtraction of the vacuum term, we get the $z$ component of the force,

$$
\begin{aligned}
F_{z}= & \sigma_{z z}=-\frac{\hbar}{4 \pi^{3}} \operatorname{Re} \int_{0}^{\infty} d \omega \int d^{2} q \frac{k_{z}}{\Delta} e^{2 i k_{z}} d\left\{q^{2}\right. \\
& \left.-\beta k q_{x}\right)^{2}\left[R_{1 p} R_{2 p}^{\prime} D_{s s}+R_{1 s} R_{2 s}^{\prime} D_{p p}\right]-\beta^{2} k_{z}^{2} q_{y}^{2}\left[R_{1 p} R_{2 s}^{\prime} D_{s p}\right. \\
& \left.\left.+R_{1 s} R_{2 p}^{\prime} D_{p s}\right]\right\}\left[1+n_{1}(\omega)+n_{2}\left(\omega^{\prime}\right)\right] .
\end{aligned}
$$

At $T_{1}=T_{2}=0 \mathrm{~K}$, Eq. (28) takes the form

$$
\begin{aligned}
F_{z}= & -\frac{\hbar}{4 \pi^{3}} \operatorname{Re}\left\{\int_{0}^{\infty} d \omega \int d^{2} q\right. \\
& \left.-\int_{-\infty}^{\infty} d q_{y} \int_{0}^{\infty} d q_{x} \int_{0}^{q_{x} V} d \omega\right\} \frac{k_{z}}{\Delta} e^{2 i k_{z} d}\left\{\left(q^{2}\right.\right. \\
& \left.-\beta k q_{x}\right)^{2}\left[R_{1 p} R_{2 p}^{\prime} D_{s s}+R_{1 s} R_{2 s}^{\prime} D_{p p}\right]-\beta^{2} k_{z}^{2} q_{y}^{2}\left[R_{1 p} R_{2 s}^{\prime} D_{s p}\right. \\
& \left.\left.+R_{1 s} R_{2 p}^{\prime} D_{p s}\right]\right\} .
\end{aligned}
$$

If in Eq. (28) one neglects the terms of the order $\beta^{2}$, then, as in the case of friction, the contributions from the waves with $p$ and $s$ polarization will be separated. In this case Eq. (28) reduces to

$$
\begin{aligned}
F_{z}= & -\frac{\hbar}{4 \pi^{3}} \operatorname{Re} \int_{0}^{\infty} d \omega \int d^{2} q k_{z}\left(\frac{1}{R_{1 p}^{-1} R_{2 p}^{\prime-1} e^{-2 i k_{z} d}-1}\right. \\
& \left.+\frac{1}{R_{1 s}^{-1} R_{2 s}^{\prime-1} e^{-2 i k_{z} d}-1}\right)\left[1+n_{1}(\omega)+n_{2}\left(\omega^{\prime}\right)\right] .
\end{aligned}
$$

If we put $V=0$ and use the Fresnel's formulas for the reflection amplitudes, then Eq. (30) reduces to the formula obtained by Lifshitz. ${ }^{4}$ Lifshitz have shown that at $T_{1}=T_{2}$ $=0 \mathrm{~K}$ it is convenient to transform $\omega$ integration along the real axis into the integral along the imaginary axis in the upper half of the complex $\omega$ plane. For a rarefied body, similarly as in Sec. III, from Eq. (28) we get the van der Waals interaction between a small particle and plane surface,

$$
\begin{aligned}
F_{z}= & -\frac{\hbar}{\pi^{2}} \operatorname{Im} \int_{0}^{\infty} d \omega \int d^{2} q \frac{k_{z}^{2} e^{2 i k_{z} d}}{\left(q^{2}-\beta k q_{x}\right)^{2}+\beta^{2} k_{z}^{2} q_{y}^{2}}\left\{\left(q^{2}\right.\right. \\
& \left.\left.-\beta k q_{x}\right)^{2}\left[R_{p} \alpha_{E}^{\prime}+R_{s} \alpha_{H}^{\prime}\right]-\beta^{2} k_{z}^{2} q_{y}^{2}\left[R_{p} \alpha_{H}^{\prime}+R_{s} \alpha_{E}^{\prime}\right]\right\}[1 \\
& \left.+n_{1}(\omega)+n_{2}\left(\omega^{\prime}\right)\right] .
\end{aligned}
$$

\section{CALCULATION OF THE RADIATIVE HEAT TRANSFER BETWEEN MOVING BODIES}

The radiative energy transfer between the bodies is determined by the ensemble average of the Poynting vector. In the case of two plane parallel surfaces the heat flux across the surface $\mathbf{1}$ is given by ${ }^{18}$

$$
\begin{aligned}
\left\langle\mathbf{S}_{1 z}(\mathbf{r})\right\rangle_{\omega} & =(c / 8 \pi)\left\langle\mathbf{E}(\mathbf{r}) \times \mathbf{B}^{*}(\mathbf{r})\right\rangle_{\omega}+\text { c.c. } \\
& =\frac{i c^{2}}{8 \pi \omega}\left\{\left\langle\mathbf{E}(\mathbf{r}) \cdot \frac{d}{d z} \mathbf{E}^{*}(\mathbf{r})\right\rangle-\text { c.c. }\right\}_{z=0} .
\end{aligned}
$$

Using Eqs. (3), (4), and (32) we get equations for the heat obtained by the body $\mathbf{1}$, which are very similar to Eqs. (21) and (22),

$$
\begin{aligned}
S_{1}= & \frac{1}{4 \pi} \int_{0}^{\infty} d \omega \int \frac{d^{2} q}{(2 \pi)^{2}} \frac{\omega}{k^{2}}\left[( k _ { z } + k _ { z } ^ { * } ) \left(\left\langle\left|w_{p}\right|^{2}\right\rangle+\left\langle\left|w_{s}\right|^{2}\right\rangle\right.\right. \\
& \left.-\left\langle\left|v_{p}\right|^{2}\right\rangle-\left\langle\left|v_{s}\right|^{2}\right\rangle\right)+\left(k_{z}-k_{z}^{*}\right)\left\langle\left(w_{p} v_{p}^{*}+w_{s} v_{s}^{*}-\text { c.c. }\right\rangle\right] .
\end{aligned}
$$

After averaging of the product of the components of the fluctuating electromagnetic field in the same way as in Sec. III we get 


$$
\begin{aligned}
S_{1}= & \frac{\hbar}{8 \pi^{3}} \int_{0}^{\infty} d \omega \int_{q<\omega / c} d^{2} q \frac{\omega}{|\Delta|^{2}}\left[\left(q^{2}-\beta k q_{x}\right)^{2}+\beta^{2} k_{z}^{2} q_{y}^{2}\right] \\
& \times\left[\left(q^{2}-\beta k q_{x}\right)^{2}\left(1-\left|R_{1 p}\right|^{2}\right)\left(1-\left|R_{2 p}^{\prime}\right|^{2}\right)\left|D_{s s}\right|^{2}+\beta^{2} k_{z}^{2} q_{y}^{2}(1\right. \\
& \left.\left.-\left|R_{1 p}\right|^{2}\right)\left(1-\left|R_{2 s}^{\prime}\right|^{2}\right)\left|D_{s p}\right|^{2}+(p \leftrightarrow s)\right]\left[n_{2}\left(\omega^{\prime}\right)-n_{1}(\omega)\right] \\
& +\frac{\hbar}{2 \pi^{3}} \int_{0}^{\infty} d \omega \int_{q>\omega / c} d^{2} q \frac{\omega}{|\Delta|^{2}}\left[\left(q^{2}-\beta k q_{x}\right)^{2}\right. \\
& \left.+\beta^{2} k_{z}^{2} q_{y}^{2}\right] e^{-2\left|k_{z}\right| d[}\left[\left(q^{2}-\beta k q_{x}\right)^{2} \operatorname{Im} R_{1 p} \operatorname{Im} R_{2 p}^{\prime}\left|D_{s s}\right|^{2}\right. \\
& \left.-\beta^{2} k_{z}^{2} q_{y}^{2} \operatorname{Im} R_{1 p} \operatorname{Im} R_{2 s}^{\prime}\left|D_{s p}\right|^{2}+(p \leftrightarrow s)\right]\left[n_{2}\left(\omega^{\prime}\right)\right. \\
& \left.-n_{1}(\omega)\right] .
\end{aligned}
$$

Equation (34) generalizes the equations for the heat transfer between two surfaces which are at the rest in the $K$ reference frame $e^{5,11}$ to the case when the surfaces are moving relative to each other. There is also the heat $S_{2}$ obtained by the body $\mathbf{2}$ in the $K^{\prime}$ reference frame. Actually, $S_{1}$ and $S_{2}$ are the same quantities, looked at from different coordinate systems. These quantities are related by the equation

$$
F_{x} V=S_{1}+S_{2} / \gamma .
$$

For the limiting case of rarefied body, similarly as in Sec. III, from Eq. (34) we get the contribution from evanescent waves to the heat adsorbed by a semi-infinite solid at $d$ $\ll \lambda_{T}$ in the $K$ reference frame for the particle-surface configuration,

$$
\begin{aligned}
S= & \frac{2 \hbar}{\pi^{2}} \int_{0}^{\infty} d \omega \int d^{2} q \frac{q \omega}{q^{2}-\beta^{2} q_{y}^{2}} e^{-2 q d}\left[n_{2}\left(\omega^{\prime}\right)-n_{1}(\omega)\right] \\
& \times\left[q^{2}\left(\operatorname{Im} R_{p} \operatorname{Im} \alpha_{E}^{\prime}+\operatorname{Im} R_{s} \operatorname{Im} \alpha_{H}^{\prime}\right)+\beta^{2} q_{y}^{2}\left(\operatorname{Im} R_{p} \operatorname{Im} \alpha_{H}^{\prime}\right.\right. \\
& \left.\left.+\operatorname{Im} R_{S} \operatorname{Im} \alpha_{E}^{\prime}\right)\right] .
\end{aligned}
$$

The heat adsorbed by a particle can be determined from Eq. (35).

\section{CALCULATION OF THE FRICTION FORCE ON A SMALL NEUTRAL PARTICle MOVING RELATIVE TO BLACK BODY RADIATION}

We consider a small neutral particle moving relative to black body radiation. We introduce two reference frame $K$ and $K^{\prime}$. The thermal radiation is in equilibrium in the $K$ reference frame, and the particle is at rest in the $K^{\prime}$ reference frames. We assume that the particle moves with velocity $V$ along the $x$ axis. The relation between the $x$ components of the momentum in the different reference frames is given by

$$
p_{x}=\left(p_{x}^{\prime}+\beta E_{0} / c\right) \gamma,
$$

where $E_{0}$ is the rest energy of the particle. The rest energy can change due to thermal radiation of the particle. From Eq. (37) we get

$$
\frac{d p_{x}}{d t}=\frac{d p_{x}^{\prime}}{d t^{\prime}}+\frac{V}{c^{2}} \frac{d E_{0}}{d t^{\prime}} .
$$

According to the Einstein's law

$$
\frac{d m_{0}}{d t^{\prime}}=\frac{1}{c^{2}} \frac{d E_{0}}{d t^{\prime}},
$$

where $m_{0}$ is the rest mast of the particle. Taking into account that

$$
\frac{d p_{x}}{d t}=\frac{d\left(m_{0} V \gamma\right)}{d t}=\frac{d m_{0}}{d t^{\prime}} V+m_{0} \frac{d(V \gamma)}{d t}
$$

from Eqs. (38)-(40) we get

$$
m_{0} \frac{d(V \gamma)}{d t}=\frac{d p_{x}^{\prime}}{d t^{\prime}} .
$$

In the rest reference frame, due to symmetry, the total radiated momentum from the dipole and magnetodipole radiation is identically zero. Thus, the change in momentum of the particle in the rest reference frame is determined by the Lorenz force $F_{x}^{\prime}$ acting on the particle from the external electromagnetic field associated with the thermal radiation observed in this reference frame. The dynamics of the particle in the $K$ reference frame is determined by the equation

$$
m_{0} \frac{d(V \gamma)}{d t}=F_{x}^{\prime}
$$

Equation (42) does not contain force from the thermal electromagnetic field radiated by the particle. Thus, from Eq. (42) it follows that, contrary to the claim of the authors of Ref. 27, the thermal radiation of the particle cannot produce any acceleration. In the $K^{\prime}$ reference frame the Lorenz force on the particle is determined by the expression ${ }^{28,29}$

$$
F_{x}^{\prime}=\frac{\partial}{\partial x^{\prime}}\left\langle\mathbf{p}_{e}^{\prime} \cdot \mathbf{E}^{\prime *}\left(\mathbf{r}^{\prime}\right)\right\rangle_{\mathbf{r}^{\prime}=\mathbf{r}_{0}^{\prime}}+\frac{\partial}{\partial x^{\prime}}\left\langle\mathbf{p}_{m}^{\prime} \cdot \mathbf{B}^{\prime *}\left(\mathbf{r}^{\prime}\right)\right\rangle_{\mathbf{r}^{\prime}=\mathbf{r}_{0}^{\prime}} .
$$

Writing the electromagnetic field as a Fourier integral, and taking into account that

$$
\begin{aligned}
& \mathbf{p}_{e}^{\prime}=\alpha_{E}\left(\omega^{\prime}\right) \mathbf{E}^{\prime}\left(\mathbf{r}_{0}^{\prime}\right) e^{-i \omega^{\prime} t^{\prime}}, \\
& \mathbf{p}_{m}^{\prime}=\alpha_{H}\left(\omega^{\prime}\right) \mathbf{B}^{\prime}\left(\mathbf{r}_{0}^{\prime}\right) e^{-i \omega^{\prime} t^{\prime}},
\end{aligned}
$$

we get

$$
\begin{aligned}
F_{x}^{\prime}= & -i \int_{\infty}^{\infty} \frac{d \omega^{\prime}}{2 \pi} \int \frac{d^{3} k^{\prime}}{(2 \pi)^{3}} k_{x}^{\prime}\left[\alpha_{E}\left(\omega^{\prime}\right)\left\langle\mathbf{E}^{\prime} \cdot \mathbf{E}^{\prime *}\right\rangle_{\omega^{\prime} \mathbf{k}^{\prime}}+\alpha_{H}\left(\omega^{\prime}\right)\right. \\
& \left.\times\left\langle\mathbf{B}^{\prime} \cdot \mathbf{B}^{\prime *}\right\rangle_{\omega^{\prime} \mathbf{k}^{\prime}}\right]=-i \int_{\infty}^{\infty} \frac{d \omega^{\prime}}{2 \pi} \int \frac{d^{3} k^{\prime}}{(2 \pi)^{3}} k_{x}^{\prime}\left[\alpha_{E}\left(\omega^{\prime}\right)\right. \\
& \left.+\alpha_{H}\left(\omega^{\prime}\right)\right]\left\langle\mathbf{E}^{\prime} \cdot \mathbf{E}^{\prime *}\right\rangle_{\omega^{\prime} \mathbf{k}^{\prime}},
\end{aligned}
$$

where we have taken into account that for plane waves $\left\langle\mathbf{E}^{\prime} \cdot \mathbf{E}^{\prime *}\right\rangle_{\omega^{\prime} \mathbf{k}^{\prime}}=\left\langle\mathbf{B}^{\prime} \cdot \mathbf{B}^{\prime *}\right\rangle_{\omega^{\prime} \mathbf{k}^{\prime}}$. When we change from the $K^{\prime}$ reference frame to the $K$ reference frame $\left\langle\mathbf{E}^{\prime} \cdot \mathbf{E}^{\prime *}\right\rangle_{\omega^{\prime} \mathbf{k}^{\prime}}$ is transformed as the energy density of a plane electromagnetic field. From the law of transformation of the energy density of a plane electromagnetic field ${ }^{30}$ we get 


$$
\left\langle\mathbf{E}^{\prime} \cdot \mathbf{E}^{\prime *}\right\rangle_{\omega^{\prime} \mathbf{k}^{\prime}}=\left\langle\mathbf{E} \cdot \mathbf{E}^{*}\right\rangle_{\omega \mathbf{k}}\left(\frac{\omega^{\prime}}{\omega}\right)^{2}
$$

According to the theory of the fluctuating electromagnetic field $^{31}$

$$
\left\langle\mathbf{E} \cdot \mathbf{E}^{*}\right\rangle_{\omega \mathbf{k}}=4 \pi^{2} \hbar k\left\{\delta\left(\frac{\omega}{c}-k\right)-\delta\left(\frac{\omega}{c}+k\right)\right\}[1+2 n(\omega)] .
$$

Taking into account the invariance of the square of the fourwave vector $(\omega / c)^{2}-k^{2}=\left(\omega^{\prime} / c\right)^{2}-k^{\prime 2}$ Eq. (46) can be rewritten in the form

$$
\begin{aligned}
\left\langle\mathbf{E} \cdot \mathbf{E}^{*}\right\rangle_{\omega \mathbf{k}}= & \frac{4 \pi^{2} \hbar k^{2}}{k^{\prime}}\left\{\delta\left(\frac{\omega^{\prime}}{c}-k^{\prime}\right)-\delta\left(\frac{\omega^{\prime}}{c}+k^{\prime}\right)\right\} \\
& \times[1+2 n(\omega)] .
\end{aligned}
$$

Substitution Eqs. (45) and (47) in Eq. (44) and integration over $\omega^{\prime}$ gives

$$
\begin{aligned}
F_{x}= & \frac{\hbar c}{2 \pi^{2}} \int d^{3} k k k_{x}\left[\operatorname{Im} \alpha_{E}(c k)+\operatorname{Im} \alpha_{H}(c k)\right] \\
& \times\left\{n\left[\gamma\left(c k+V k_{x}\right)\right]-n\left[\gamma\left(c k-V k_{x}\right)\right]\right\},
\end{aligned}
$$

where we have omitted index of prime and have taken into account that $\omega=\left(\omega^{\prime}+k_{x}^{\prime} V\right) \gamma$. Introducing the new variable $\omega=c k$, Eq. (48) can be transformed to the form

$$
\begin{aligned}
F_{x}= & \frac{2 \hbar}{\pi c^{2}} \int_{0}^{\infty} d \omega \omega^{2} \int_{0}^{\omega / c} d k_{x} k_{x}\left[\operatorname{Im} \alpha_{E}(\omega)+\operatorname{Im} \alpha_{H}(\omega)\right] \\
& \times\left\{n\left[\gamma\left(c k+V k_{x}\right)\right]-n\left[\gamma\left(c k-V k_{x}\right)\right]\right\} .
\end{aligned}
$$

Equation (49) generalizes the result obtained in Ref. 22 to the case of large velocities and includes the contribution from the magnetic moment. For metallic particles the contribution from the magnetic moment exceeds substantially the contribution from the electric dipole moment. At small velocities $F_{x}=-\Gamma V$, where

$$
\Gamma=\frac{4 \hbar}{3 \pi c^{5}} \int_{0}^{\infty} d \omega\left(-\frac{\partial n}{\partial \omega}\right) \omega^{5}\left[\operatorname{Im} \alpha_{E}(\omega)+\operatorname{Im} \alpha_{H}(\omega)\right] .
$$

For metals with $4 \pi \sigma \gg k_{B} T / \hbar$ and for $c \sqrt{2 \pi \sigma k_{B} T} \gg R$, where $\sigma$ is the conductivity, from Eqs. (25) and (26) we get

$$
\begin{gathered}
\operatorname{Im} \alpha_{E}(\omega) \approx R^{3} \frac{3 \omega}{4 \pi \sigma}, \\
\operatorname{Im} \alpha_{H}(\omega)=\frac{4 \pi \sigma \omega R^{5}}{30 c^{2}} .
\end{gathered}
$$

Setting the friction coefficient $\Gamma$ to $m_{0} / \tau$, where $\tau$ the relaxation time, and using $m_{0}=4 \pi R^{3} \rho / 3$, from Eqs. (50)-(52) we get

$$
\tau_{e}^{-1} \approx 10^{2} \frac{\hbar}{\rho \lambda_{T}^{5}} \frac{k_{B} T}{\hbar \sigma}
$$

$$
\tau_{m}^{-1} \approx 10^{2} \frac{\hbar R}{\rho \lambda_{T}^{6}} \frac{\sigma R}{c},
$$

where $\tau_{e}^{-1}$ and $\tau_{m}^{-1}$ are the contributions to the friction from the electric dipole and magnetic moments, respectively. For $T=300 \mathrm{~K}, \rho \approx 10^{4} \mathrm{~kg} / \mathrm{m}^{3}$, and $\sigma \approx 10^{18} \mathrm{~s}^{-1}$ from Eqs. (51) and (54) we get $\tau_{e} \sim 10^{16} \mathrm{~s}$ and $\tau_{m} \sim 10^{12} \mathrm{~s}$. When the conductivity decreases $\tau_{e}$ also decreases and reaches minimum at $2 \pi \sigma \approx k_{B} T / \hbar$. At $T=3000 \mathrm{~K}$ this minimum corresponds to about a day $\left(\tau_{e}^{\min } \approx 10^{5} \mathrm{~s}\right)$. In Ref. 22 the same relaxation time was obtained for $\mathrm{Ba}^{+}$.

\section{CONCLUSION}

In this paper within the framework of unified approach we have calculated the Casimir-Lifshitz interaction, the van der Waals friction force, and the radiative heat transfer at nonequilibrium conditions when the interacting bodies are at different temperatures and when they move relative to each other with the arbitrary velocity $V$. In comparison with the existing literature we have studied more general nonequilibrium conditions. Our study was focused on the surfacesurface and surface-particle configurations. We have found the exact solution of problem about the determination of the fluctuating electromagnetic field in the vacuum gap between two flat parallel surfaces moving relative to each other. Knowing the electromagnetic field we have calculated the Maxwell stress tensor and the Poynting vector which determine the friction, conservative forces, and the heat transfer between the solids, respectively. For the heat transfer and the conservative force our treatment generalizes the results obtained for bodies at rest to the case of bodies which move relative to each other. The velocity dependence of the considered phenomena can be strong if the surfaces of the bodies can support localized surface modes such as coupled plasmon-polaritons, phonon-polaritons, or adsorbate vibrational modes. In this case, when interaction is determined by the resonances, connected with these surface modes, Doppler shift will lead to the displacement of resonances relative to each other. If resonances are sufficiently sharp, then it will lead to the strong dependence on the velocity. This effect can be used for the precision determination of energy of the surface modes. We have shown that relativistic effects produce a mixing of the $s$ - and $p$-wave contributions to the forces and the heat transfer. This relativistic effect is of the order $(V / c)^{2}$. If one neglects by terms of order $(V / c)^{2}$, the different polarizations will contribute to the forces and the heat transfer separately. From the limit when one of the bodies is rarefied, we have calculated the interaction force and the heat transfer for a small particle outside a flat surface. For a particle we have taken into account the contribution to the forces and the heat transfer from the electric dipole and magnetic moments. For metallic particles we have found that the contribution to the friction from the magnetic moment exceeds the contribution from the electric dipole moment by several orders of magnitude. We have presented a relativistic theory of the friction force acting on a particle moving relative to the black body radiation. We have shown that the thermal radiation of the particle does not produce any acceleration and 
that the friction force acting on the particle is determined by external electromagnetic field associated with the black body radiation. For a metallic particle moving relative to the black body radiation the friction force increases initially when the conductivity decreases, reaches maximum at $2 \pi \sigma \approx \omega_{T}$, and then decreases.

\section{ACKNOWLEDGMENTS}

A.I.V. acknowledges financial support from the Russian Foundation for Basic Research (Grant No. 08-02-00141-a) and DFG.
*Corresponding author; alevolokitin@yandex.ru

${ }^{1}$ S. M. Rytov, Theory of Electrical Fluctuation and Thermal Radiation (Academy of Science of USSR, Moscow, 1953).

${ }^{2}$ M. L. Levin and S. M. Rytov, Theory of Equilibrium Thermal Fluctuations in Electrodynamics (Science, Moscow, 1967).

${ }^{3}$ S. M. Rytov, Yu. A. Kravtsov, and V. I. Tatarskii, Principles of Statistical Radiophyics (Springer, New York, 1989), Vol. 3.

${ }^{4}$ E. M. Lifshitz, Zh. Eksp. Teor. Fiz. 29, 94 (1955) [Sov. Phys. JETP 2, 73 (1956)].

${ }^{5}$ D. Polder and M. Van Hove, Phys. Rev. B 4, 3303 (1971).

${ }^{6}$ A. I. Volokitin and B. N. J. Persson, J. Phys.: Condens. Matter 11, 345 (1999); Phys. Low-Dimens. Struct. 7/8, 17 (1998).

${ }^{7}$ M. Antezza, L. P. Pitaevskii, S. Stringari, and V. B. Svetovoy, Phys. Rev. A 77, 022901 (2008).

${ }^{8}$ M. Antezza, L. P. Pitaevskii, and S. Stringari, Phys. Rev. Lett. 95, 113202 (2005).

${ }^{9}$ M. Antezza, L. P. Pitaevskii, S. Stringari, and V. B. Svetovoy, Phys. Rev. Lett. 97, 223203 (2006).

${ }^{10}$ J. B. Pendry, J. Phys.: Condens. Matter 11, 6621 (1999).

${ }^{11}$ A. I. Volokitin and B. N. J. Persson, Phys. Rev. B 63, 205404 (2001); Phys. Low-Dimens. Struct. 5/6, 151 (2001).

${ }^{12}$ A. I. Volokitin and B. N. J. Persson, Phys. Rev. B 69, 045417 (2004).

${ }^{13}$ A. I. Volokitin and B. N. J. Persson, JETP Lett. 78, 457 (2003).

${ }^{14}$ J. P. Mulet, K. Joulin, R. Carminati, and J. J. Greffet, Appl. Phys. Lett. 78, 2931 (2001).

${ }^{15}$ J. P. Mulet, K. Joulain, R. Carminati, and J. J. Greffet, Microscale Thermophys. Eng. 6, 209 (2002).

${ }^{16}$ A. Kittel, W. Müller-Hirsch, J. Parisi, S. A. Biehs, D. Reddig, and M. Holthaus, Phys. Rev. Lett. 95, 224301 (2005).

${ }^{17}$ K. Joulain, J. P. Mulet, F. Marquier, R. Carminati, and J. J. Greffet, Surf. Sci. Rep. 57, 59 (2005).

${ }^{18}$ A. I. Volokitin and B. N. J. Persson, Rev. Mod. Phys. 79, 1291 (2007).

${ }^{19}$ A. I. Volokitin and B. N. J. Persson, J. Phys.: Condens. Matter 13, 859 (2001).

${ }^{20}$ A. I. Volokitin and B. N. J. Persson, Phys. Rev. Lett. 91, 106101 (2003).

${ }^{21}$ A. I. Volokitin and B. N. J. Persson, Phys. Rev. B 68, 155420 (2003).

${ }^{22}$ V. Mkrtchian, V. A. Parsegian, R. Podgornik, and W. M. Saslow, Phys. Rev. Lett. 91, 220801 (2003).

${ }^{23}$ P. O. Chapuis, M. Laroche, S. Volz, and J. J. Greffet, Phys. Rev. B 77, 125402 (2008).

${ }^{24}$ J. B. Pendry, J. Phys.: Condens. Matter 9, 10301 (1997).

${ }^{25}$ L. D. Landau and E. M. Lifshitz, Electrodynamics of Continuous Media (Pergamon, Oxford, 1960).

${ }^{26}$ J. Schwinger, L. DeRaad, and K. Milton, Ann. Phys. (N.Y.) 115 , 1 (1978).

${ }^{27}$ G. V. Dedkov and A. A. Kyasov, Phys. Lett. A 339, 212 (2005).

${ }^{28}$ A. I. Volokitin and B. N. J. Persson, Phys. Rev. B 65, 115419 (2002).

${ }^{29}$ G. V. Dedkov and A. A. Kyasov, Tech. Phys. 53, 389 (2008).

${ }^{30}$ L. D. Landau and E. M. Lifshitz, The Classical Theory of Field (Pergamon, Oxford, 1975).

${ }^{31}$ E. M. Lifshitz and L. P. Pitaevskii, Statistical Physics (Pergamon, Oxford, 1980), Pt. 2. 